\title{
Assessment of different topographic corrections in AWiFS satellite imagery of Himalaya terrain
}

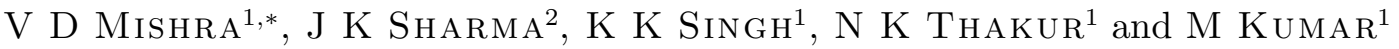 \\ ${ }^{1}$ Snow and Avalanche Study Establishment, Defence Research and Development Organisation, \\ Chandigarh 160 036, India. \\ ${ }^{2}$ Rayat Institute of Engineering 8 Information Technology, Nawanshahr, Punjab 144 533, India. \\ *e-mail: vd_mishra@rediffmail.com
}

The influence of topographic effects in optical satellite imagery is not investigated very extensively in the Himalayan terrain. The topographic variability causes a problem of differential illumination due to steep and varying slopes in rugged Himalayan terrain. Therefore, topographic corrections are essential for qualitative and quantitative analysis of snow cover applications. The present paper discusses the implementation of different topographic correction models on AWiFS sensor onboard IRS P6 satellite images and the qualitative and quantitative comparative analysis in detail. Both the Lambertian and non-Lambertian assumptions have been considered in the present analysis with the aim to explore best suitable empirical model for rugged terrain. The main topographic methods implemented are:

- C-correction

- Minneart corrections

- Civco's modified version of cosine correction

- two-stage normalization and

- slope matching technique.

Lambertian assumptions are found to be very unrealistic over Himalayan terrain as these lead to either underestimation or overestimation of physical parameters significantly both on sunlit slopes as well as the slopes away from the Sun. This problem is overcome by considering non-Lambertian assumption. Minneart constant and C-correction coefficients for all AWiFS satellite bands are estimated using regression analysis. All the results due to topographic effects are investigated qualitatively and quantitatively using four criteria namely visual analysis, validation with field measurements (in-situ observations), spectral reflectance of training samples of snow on the south and north aspects and graphically. The visual analysis confirms the minimization of three dimensional relief effects in two-stage normalization and slope matching methods and retrieves some of the information under mountain shadow. Due to the very bright surface of snow fields there is likely to be more diffuse reflected light in these areas than over darker vegetated surfaces. The qualitative analysis in other methods does not extract any information on shady slopes. The quantitative validation of topographic results in satellite imagery with in-situ observations shows underestimation of spectral reflectance of snow significantly except for slope matching technique. It is also apparent that although all the topographic methods correct the reflectance of training snow samples on the south and north aspects but most acceptable values are achieved using slope matching. The results obtained from graphical analysis reveal that mean reflectance after all topographic corrections are independent of illumination. This study also suggests that the suitability of

Keywords. Topographic normalization; C-corrections; Minneart constant; slope match. 
topographic models can not be concluded as successful based on single criterion. Slope matching technique is the only technique which satisfies all the four criteria successfully and produces the best result for Himalayan terrain.

\section{Introduction}

Optical satellite imagery in mountainous terrain is affected due to sharp variations in the topographic parameters such as altitude, slope and aspect. This in turn has a significant influence on the qualitative and quantitative estimation of snow and other land cover characteristics. The variations of the topographic parameters affect the variations in the brightness of the satellite images having different spectral and spatial resolution. The topographic corrections or topographic normalization refers (Riano et al 2003) to the compensation of differential solar illumination due to rugged shape of the mountainous terrain. Differential illumination results in considerable variation in the spectral characteristics of similar snow and other land covers. Sun-facing illuminated slopes (south aspect) show more than expected spectral radiance or reflectance, whereas the effect is opposite in shaded relief area (north aspect) (Riano et al 2003). These differential illumination effects in satellite imagery will suppress the maximum information on the north-facing slopes, thereby adversely affecting the results of various quantitative methods of snow cover monitoring namely classification, temporal change analysis and other hazard related information. The direct solar irradiance on any inclined pixel will be different as compared to flat surface on the satellite images. Therefore, effective removal or minimization of terrain effects is essential for snow cover monitoring, e.g., corrected spectral reflectance (Xin et al 2002), estimation and mapping broadband albedo (Duguay and LeDrew 1991; Song and Gao 1999), spectral mixing (Romanov et al 2003; Haertel and Shimabukuro 2005), energy balance and melt processes (Brun et al 1989; Duguay 1993; Upadhyay 1995; Mukkoth 2004), temporal and spectral changes (Davis et al 1993), spatial patterns of temperature (Deems 2001) in mountainous area.

A wide range of commonly used topographic correction methods have been reported in the literature (Minneart 1941; Smith et al 1980; Teillet et al 1982; Dozier 1984; Hall et al 1988; Leprieur et al 1988; Civco 1989; Colby 1991; Tokola et al 2001). These are cosine method, C-correction, Minneart correction and the two-stage normalization, etc. Generally, these methods have been used for qualitative analysis and mapping of vegetation types and forest cover. In very few cases, some of these topographic correction methods (Dozier
1984; Hall et al 1988, Civco 1989) have been used for snow applications in Himalayan terrain but the results are not validated with in-situ observations. Recently Mishra et al (2008) has reported that these few algorithms give erroneous results for Himalayan terrain and underestimate snow spectral reflectance significantly when validated with in-situ observations. Snow cover analysis using all models is not explored extensively for rugged and complex Himalayan terrain. In the present study, all these methods are implemented on medium spatial resolution $(56 \mathrm{~m})$ AWiFS satellite images and the influence on reflectance results are discussed. Newly reported slope matching technique (Nichol et al 2006) is also implemented and comparative analysis with other methods is discussed in detail. This new method is reported to produce encouraging results for high resolution satellite imagery of IKONOS for vegetation analysis and for low sun elevation angle. It is necessary to validate the efficacy of slope matching technique for medium resolution images with varying solar geometry (solar zenith, azimuth and illumination).

The main objective of the present paper is:

- to implement all topographic models for qualitative and quantitative assessment for carrying out comparative analysis of snow reflectance estimation on medium resolution satellite imageries of Western Himalaya obtained from AWiFS sensors onboard IRS-P6

- visual analysis

- validation of terrain corrected satellite estimated reflectance by all theses methods with field measurements

- testing of topographically corrected results for spectral reflectance of snow samples on the south and north aspects

- graphical analysis after topographic corrections and

- to find the best suitable method for topographic corrections for Western Himalaya.

Image based atmospheric corrections (Chavez 1989; Song et al 2001) for path radiance and Rayleigh scattering is performed first for initial estimation of reflectance without considering topographic influences. The details of the implementation of these corrections on AWiFS satellite imageries are given by Mishra et al (2008). 


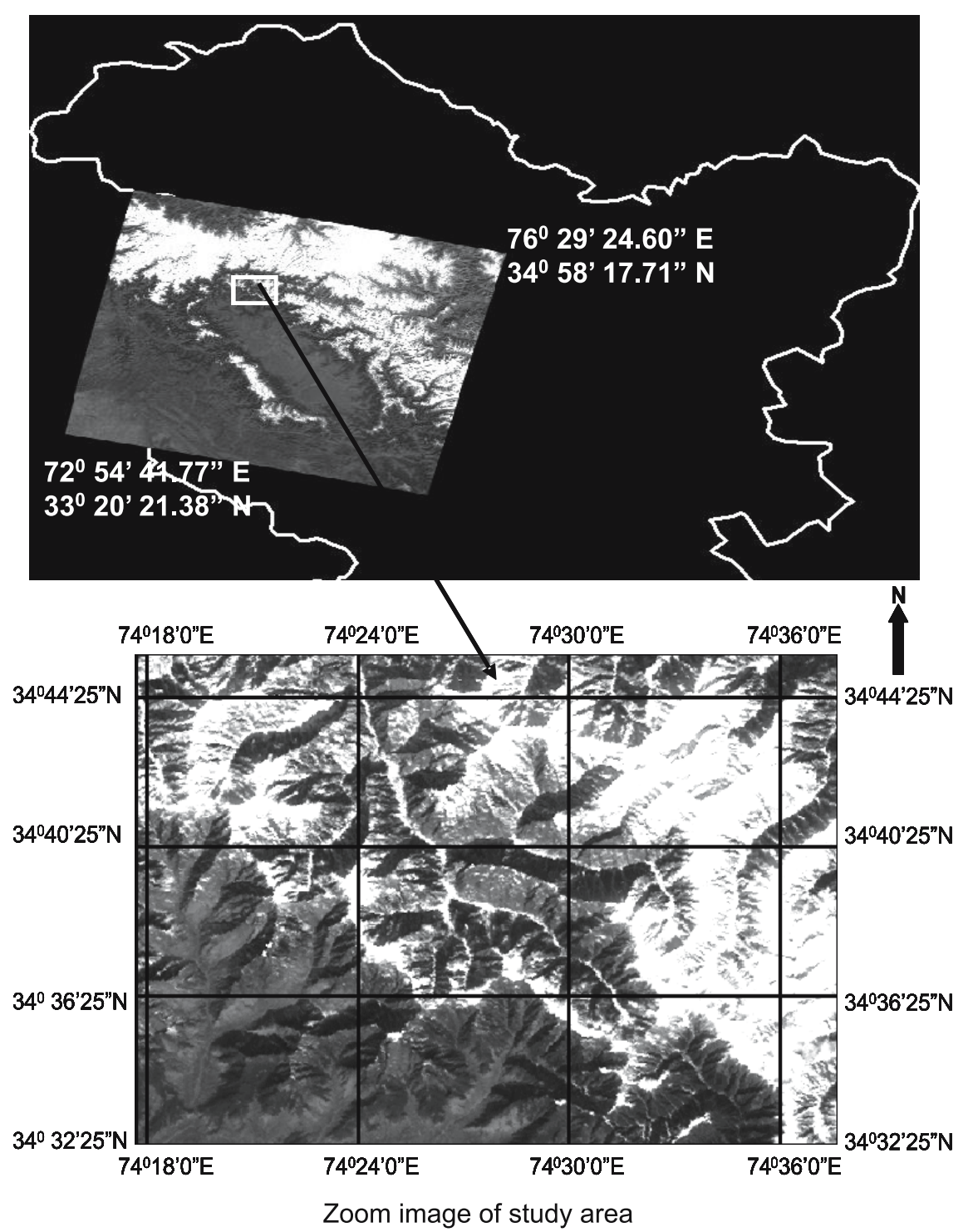

Figure 1. Top: AWiFS satellite image of Western Himalaya and bottom: zoom image of the study area shown with white rectangle.

\section{Methodology}

\subsection{Study area}

The study area lies in the north-east tip of Samsabari range in Jammu and Kashmir (J\&K) region of Western Himalaya as shown in figure 1. Average elevation of the area is in between 3000 and $3500 \mathrm{~m}$ and slopes vary from $0^{\circ}$ to $55^{\circ}$. Majority of the slopes have eastern and southern aspects. The area is densely forested in between 2400 and $3100 \mathrm{~m}$ altitude. Beyond $3100 \mathrm{~m}$ forest is scanty; however, below $2400 \mathrm{~m}$ area is habitated. Generally, this area receives moderate-toheavy snowfall above $2400 \mathrm{~m}$ with intense wind activity. The flow chart of detailed methodology of processing steps is given in figure 2 .

\subsection{Satellite dataset}

Three almost cloud free satellite images recorded by AWiFS sensor onboard IRS-P6 (RESOURCESAT-I) on 11 December 2005, 18 January 2005 and 21 February 2005 are used in the present work. The selection of these three images is mainly to facilitate:

- carrying out analysis of the topographic corrections for different solar zenith, azimuth angles and illumination

- validation of spectral reflectance with satellite estimated results as the field observations were recorded in near real time at the time of satellite pass on 18 January and 21 February 2005. 


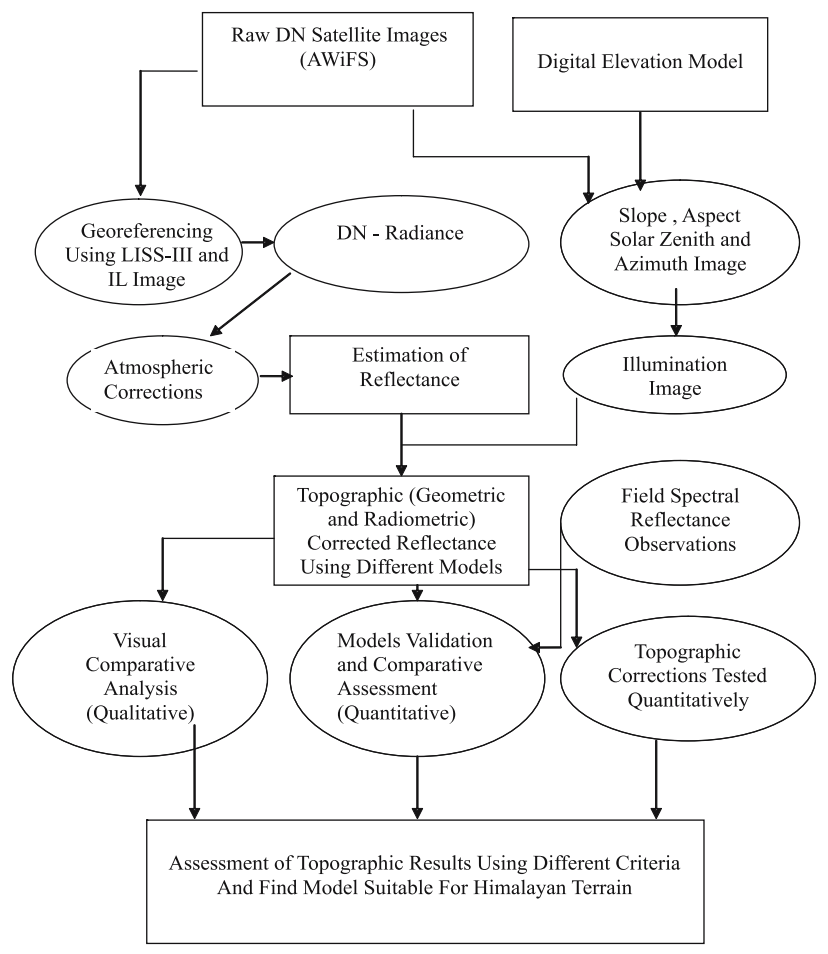

Figure 2. Flow chart showing methodology and processing steps for different topographic models.

The main AWiFS sensor specifications in different spectral bands are given in table 1 .

\subsection{Digital elevation model}

A digital elevation model (DEM) of the study area was generated using 1:50,000 toposheet at $40 \mathrm{~m}$ contour level. The non-linear interpolation function is used for 3-D surface generation. The grid resolution of the DEM is $6 \mathrm{~m}$ which was further re-sampled at $56 \mathrm{~m}$ to bring it equal to the grid resolution of AWiFS satellite images. DEM was further used to generate terrain parameters for topographic models using ERDAS Imagine 8.7.

\subsection{Geometric corrections}

The accuracy of geo-referencing is very important for topographic corrections. All the satellite images were geo-referenced with respect to LISS-III image rectified using 1:50,000 scale map. The topographic corrections require accurate matching of satellite images with digital elevation model. This is a challenging task for the successful implementation of topographic models in rugged Himalayan terrain which otherwise leads to erroneous results. The geo-referencing of AWiFS images with LISS-III seldom matches completely with the digital elevation model due to different amount of shadow in satellite data arising from differential illumination, solar zenith and azimuth angle. It is not convenient to check the matching of satellite images with digital elevation model (DEM) directly. In order to avoid any mismatch of the satellite images with the digital elevation model, it was further geo-referenced and matched perfectly with the illumination image generated using the digital elevation model. The cosine of $(i)$ gives the illumination (IL), where $i$ is local incidence angle. The illumination model is described in section 3.1. The nearest neighbour resampling method was used and the geolocation error of the model is about half a pixel.

\subsection{Topographically uncorrected surface reflectance}

Image based atmospherically corrected true spectral reflectance on the tilted surface, $R_{\lambda T}$, from the sensor radiance, $L_{\lambda}$, is obtained using the following model (Song et al 2001; Pandya et al 2002).

$$
R_{\lambda T}=\frac{\pi\left(L_{\lambda}-L_{p}\right) d^{2}}{t_{v}\left(E_{0} \cos \theta_{z} t_{z}+E_{d}\right)}
$$

where $L_{p}$ is the path radiance in $\mathrm{mW} / \mathrm{cm}^{2} / \mathrm{sr} / \mu \mathrm{m}$ and estimated according to (Chavez 1989, 1996), $d$ is the Earth-Sun distance in astronomical units and calculated using the approach of Van (1996). $E_{0}$ is the bandpass exoatmospheric spectral irradiance (table 1), $E_{d}$ is the downwelling spectral irradiance at the surface due to diffused radiation and assumed to be equal to zero according to

Table 1. Salient specifications of AWiFS sensor.

\begin{tabular}{|c|c|c|c|c|c|}
\hline $\begin{array}{l}\text { Spectral } \\
\text { bands } \\
(\mathrm{nm})\end{array}$ & $\begin{array}{l}\text { Spatial } \\
\text { resolution } \\
\text { at Nadir } \\
(\mathrm{m})\end{array}$ & $\begin{array}{c}\text { Quantization } \\
\text { (bit) }\end{array}$ & $\begin{array}{l}\text { Minimum } \\
\text { radiance } \\
\left(\mathrm{mW} \mathrm{cm}^{-2}\right. \\
\left.\mathrm{Sr}^{-1} \mu \mathrm{m}^{-1}\right)\end{array}$ & $\begin{array}{l}\text { Maximum } \\
\text { radiance } \\
\left(\mathrm{mW} \mathrm{cm}^{-2}\right. \\
\left.\mathrm{Sr}^{-1} \mu \mathrm{m}^{-1}\right)\end{array}$ & $\begin{array}{c}\text { Mean solar exo- } \\
\text { atmospheric } \\
\text { spectral } \\
\text { irradiance } \\
\left(\mathrm{mW} \mathrm{cm}^{-2} \mu \mathrm{m}^{-1}\right)\end{array}$ \\
\hline $520-590$ & 56 & 10 & 0 & 52.34 & 185.3218 \\
\hline $620-680$ & 56 & 10 & 0 & 40.75 & 158.042 \\
\hline $770-860$ & 56 & 10 & 0 & 28.425 & 108.357 \\
\hline $1550-1700$ & 56 & 10 & 0 & 4.645 & 23.786 \\
\hline
\end{tabular}


Chavez (1989), $\theta_{z}$ is the solar zenith angle and calculated (Kasten 1962) for each pixel of the study area, $t_{v}$ is the atmospheric transmittance along the path from ground surface to sensor and $t_{z}$ is the atmospheric transmittance along the path from Sun to ground.

\section{Review of topographic models}

\subsection{Cosine correction}

This is the most widely used common method for topographic correction based on trigonometric approach and assumes Lambertian reflectance characteristics of the land cover. The cosine method for the Lambertian assumption (Teillet et al 1982; Dozier 1984) which is later modified by Civco (1989) is given as:

$$
R_{\lambda H}=R_{\lambda T}\left(\frac{\cos \theta_{z}}{\cos i}\right),
$$

where $R_{\lambda H}$ is the spectral reflectance for horizontal surface, $R_{\lambda T}$ is spectral reflectance observed over the inclined terrain; $\cos i$ is illumination $(I L)$ and calculated using equation proposed by (Civco 1989; Kawata et al 1995; Riano et al 2003):

$$
\begin{aligned}
I L= & \cos i=\cos \left(\theta_{p}\right) \cos \left(\theta_{z}\right) \\
& +\sin \left(\theta_{p}\right) \sin \left(\theta_{z}\right) \cos \left(\Phi_{a}-\Phi_{0}\right),
\end{aligned}
$$

where $i$ is the local incidence angle and can be defined as the angle between the direct solar rays and normal to the surface, $\theta_{p}$ is the slope of the surface, $\Phi_{a}$ and $\Phi_{0}$ are the aspects of the surface and solar azimuth angle respectively.

\section{$3.2 C$-correction}

This correction is based on non-Lambertian assumption of the surface with modification of cosine correction developed by Teillet et al (1982). A quantity, $c_{\lambda}$, is introduced in equation (2) defined as:

$$
R_{\lambda H}=R_{\lambda T}\left(\frac{\cos \theta_{z}+c_{\lambda}}{\cos i+c_{\lambda}}\right)
$$

where $c_{\lambda}\left(=b_{\lambda} / m_{\lambda}\right)$ is a quotient between intercept $\left(b_{\lambda}\right)$ and slope $\left(m_{\lambda}\right)$ in different spectral bands. It is estimated using regression equation assuming the linear relationship between original uncorrected reflectance and $\cos i$ as $R_{\lambda T}=m_{\lambda} \cos i+b_{\lambda}$.

\subsection{Minneart correction}

Minneart correction function is based on the assumption of non-Lambertian surface reflectance characteristics and reported to be very effective in rugged terrain (Colby 1991). This function was developed by Minneart (1941) who formulated a semi-empirical equation to evaluate the roughness of the moon's surface. The function has been used for photometric analysis of lunar surfaces (Holben and Justice 1980) and implemented for topographic corrections as given below:

$$
R_{\lambda H}=R_{\lambda T}\left(\frac{\cos \theta_{z}}{\cos i}\right)^{k(\lambda)}
$$

where $k(\lambda)$ is Minneart constant and depends on the type of surface and spectral wavelength bands. It varies from zero (ideally non-Lambertian surface) to one (perfect Lambertian surface). The value of $k(\lambda)$ can be determined by linearizing the equation (5), which then becomes as given below:

$$
\ln \left(R_{\lambda T}\right)=\ln \left(R_{\lambda H}\right)+k(\lambda)^{*} \ln \left(\frac{\cos i}{\cos \theta_{z}}\right) .
$$

Equation (5) was further modified by some authors (Colby 1991) to include the slope of the terrain as follows:

$$
\begin{gathered}
R_{\lambda H}=R_{\lambda T} \cos \theta_{p}\left(\frac{\cos \theta_{z}}{\cos i \cos \theta_{p}}\right)^{k(\lambda)} . \\
3.4 \begin{array}{c}
\text { Single and two-stage normalization } \\
\text { (mean illumination method) }
\end{array}
\end{gathered}
$$

Several authors have reported (Holben and Justice 1980; Duguay and Ledrew 1992; Meyer et al 1993) that cosine correction in equation (2) overcorrects the image, mainly in areas of low IL because $\cos i \leq 0$. Initially, an improved version of equation (2) has been proposed by Civco (1989). The model is wavelength dependent which considers the mean IL conditions and single stage normalization. The image reflectance values are normalized using the illumination model. The illumination values ranging from -1 to +1 are scaled to a range of 0-255 (Nichol et al 2006) and then single stage normalization is computed using equation (8) as:

$$
R_{n \lambda i j}=R_{\lambda i j}+\left[\frac{R_{\lambda i j}\left(\langle\cos i\rangle-\cos _{i j} i\right)}{\langle\cos i\rangle}\right],
$$


where $R_{n \lambda i j}$ is the normalized reflectance values for image pixel $i j$ in wave band $\lambda ; R_{\lambda i j}=R_{\lambda T}$ is the uncorrected original reflectance on the tilted surface for image pixel $i j$ in wave band $\lambda ;\langle\cos i\rangle$ is the mean value of scaled (0-255) IL of the entire image and $\cos _{i j} i$ is IL image for each pixel $i j$ of the study area. Civco (1989) later observed that the first stage normalization in equation (8) retain the mean spectral response of the image data while reducing the variance in similar land cover classes. He further observed that the topographic effect was not completely removed in the low illumination area on north facing slopes and remained dark as compared to south facing slopes.

The remaining topographic effect was further removed by Civco (1989) from the satellite images in second stage normalization by introducing an empirically estimated calibration coefficient, $C$. The coefficient $C$ is estimated using spectral response from all the samples on the slopes facing to and away from the Sun using first stage correction (equation 8) and uncorrected reflectance image using equation (9).

$$
\begin{aligned}
C_{\lambda}= & {\left[\left(\mu_{\lambda}-N_{\lambda}\right) /\left(\left(\mu_{\lambda}-N_{\lambda}\right)-\left(\mu_{\lambda}-N_{\lambda}^{\prime}\right)\right)\right] } \\
& +\left[\left(\mu_{\lambda}-S_{\lambda}\right) /\left(\left(\mu_{\lambda}-S_{\lambda}\right)-\left(\mu_{\lambda}-S_{\lambda}^{\prime}\right)\right)\right],
\end{aligned}
$$

where $C_{\lambda}$ is the correction coefficient for different spectral wave band $\lambda, \mu_{\lambda}$ is the mean original uncorrected reflectance of the entire image in different spectral bands, $\lambda, N_{\lambda}$ is the mean reflectance value on the slopes facing away from the Sun (north aspect) in uncorrected image data, $N_{\lambda}^{\prime}$ is the mean reflectance values on the slopes facing away from the Sun after first stage normalization. $S_{\lambda}$ is the mean reflectance value on slopes facing to the Sun (south aspect) in uncorrected image data and $S_{\lambda}^{\prime}$ is the mean reflectance value on slopes facing to the Sun after single stage normalization.

The second stage normalization correction is then applied for the uncorrected original reflectance image using the following equation:

$$
R_{n \lambda i j}=R_{\lambda i j}+\left[R_{\lambda i j} \frac{\left(\langle\cos i\rangle-\cos _{i j} i\right)}{\langle\cos i\rangle}\right] X_{\lambda} .
$$

\subsection{Slope matching technique}

Nichol et al (2006) observed that the equation (10) developed by Civco (1989) using equations $(8,9)$ is inadequate for topographic corrections on the satellite images due to two reasons: (i) it does not equalize the reflectance or radiance values between the south aspect (sun facing slopes) and north aspect (shady slopes) and consider mean IL $(\langle\cos i\rangle)$ of an entire image for normalization and (ii) coefficient $C_{\lambda}$, does not determine the spectral reflectance difference between south and north aspects because north aspect is not normalized with respect to spectral response of main cover type of the south aspect. Nichol et al (2006) modified equation (10) and suggested that it is more important to make the extent of corrections equal to the range of reflectance values $\left(R_{\max }-R_{\min }\right)$ i.e., maximum difference of reflectance between south and north aspects. Considering the mean IL $(\langle\cos i\rangle)$ of an entire image in equation (10) does not retrieve the information under mountain shadow. Therefore, DN values in satellite image are normalized to the maximum illumination levels of pixels on the south aspect as compared to mean IL values of an entire image. In the analysis of Nichol et al (2006) the slope matching technique was proposed for a vegetated study area, and implemented for satellite images of very high resolution of IKONOS acquired for sun elevation angle of $38-42^{\circ}$. The application of this new method is not explored for medium and coarser resolution satellite data. The new slope matching method is necessary to be implemented and tested for medium resolution AWiFS images (56 m spatial resolution) of Himalaya for snow cover applications for varying solar elevation angles, solar azimuth and illumination. DN (0-255) image is used as an input in the reported slope matching method. We have considered reflectance image as an input in slope matching method because the same DN (0-1023) values on different AWiFS scenes are likely to correspond to different reflectances (Hall et al 1995). It is important to express the data in physical units, for example reflectance. The image normalization also performs in two stages. The first stage of the two-stage normalization by slope matching technique is adapted as follows:

The required equation for the first stage normalization is given as:

$$
\begin{aligned}
R_{n \lambda i j}= & R_{\lambda i j}+\left(R_{\max }-R_{\min }\right) \\
& \times \frac{\left(\langle\cos i\rangle_{s}-\cos _{i j} i\right)}{\langle\cos i\rangle_{s}},
\end{aligned}
$$

where notations are same as in equation (8), except $\langle\cos i\rangle_{s}$, which is the mean value of illumination on the south aspect rather than the overall mean of the entire image. $R_{\max }$ and $R_{\min }$ are the maximum 
and minimum reflectance values of the land cover in uncorrected image. Therefore, the advantage of this method is that reflectance values in the corrected image are normalized to the mean illumination level of pixels on the sunny aspect rather than the overall mean illumination value of the entire image (Nichol et al 2006).

The correction coefficient, $C_{\lambda}$, in this new method is also different from that of Civco (1989) adapted in equation (9). $C_{\lambda}$ in different wavelength bands, $\lambda$, is determined using the difference of reflectance values between sunlit and shady slopes with the uncorrected and first stage normalized image using the following equation:

$$
C_{\lambda}=\frac{S_{\lambda}^{\prime}-N_{\lambda}}{N_{\lambda}^{\prime}-N_{\lambda}},
$$

where $S_{\lambda}^{\prime}$ is the mean reflectance value on sunlit slopes after first stage normalization, $N_{\lambda}$ is the mean reflectance value on shady slopes in uncorrected image and $N_{\lambda}^{\prime}$ is the mean reflectance value on shady slope after first stage normalization.

The final form of slope matching method for topographic corrections can be expressed as:

$$
\begin{aligned}
R_{n \lambda i j}= & R_{\lambda i j}+\left(R_{\max }-R_{\min }\right) \\
& \times\left[\frac{\left(\langle\cos i\rangle_{s}-\cos _{i j} i\right)}{\langle\cos i\rangle_{s}}\right] C_{\lambda} .
\end{aligned}
$$

\section{Results}

4.1 Solar zenith, solar azimuth, terrain slope and aspect and illumination

The solar geometry parameters, viz., solar zenith angle and solar azimuth angle, were computed for each pixel using the equations given in the literature (Kasten 1962; Teillet et al 1982). The mean solar zenith angle for three AWiFS images of 11 December 2005, 18 January 2005 and 21 February 2005 are estimated to be $58.7^{\circ}, 55.7^{\circ}$ and $45^{\circ}$ respectively. The topographic correction models are implemented for these images in order to test the suitability of the models for three different sun elevation angles varying approximately from $31^{\circ}$ (low sun elevation) to $45^{\circ}$ (high sun elevation). Although mean solar azimuth angle does not vary significantly and is estimated to be $157.5^{\circ}$, $158.6^{\circ}$ and $156.7^{\circ}$ respectively for the three AWiFS passes as mentioned above. Terrain slope, aspect and IL are the three important inputs required in topographic correction models for Himalayan (a)

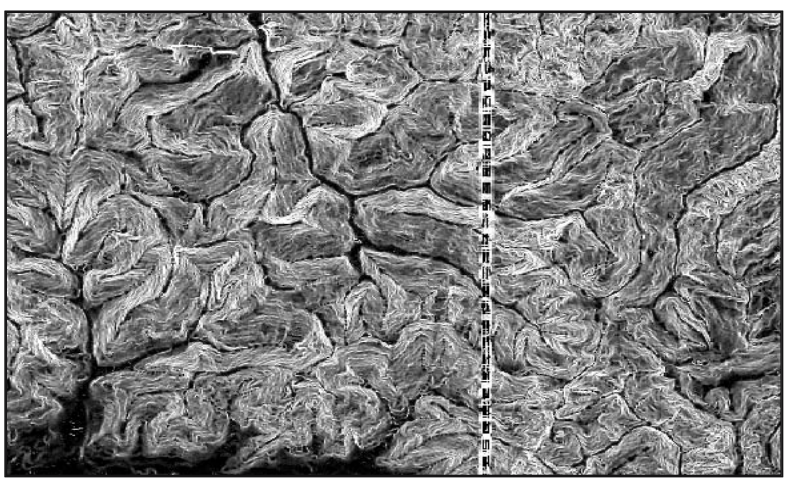

(b)

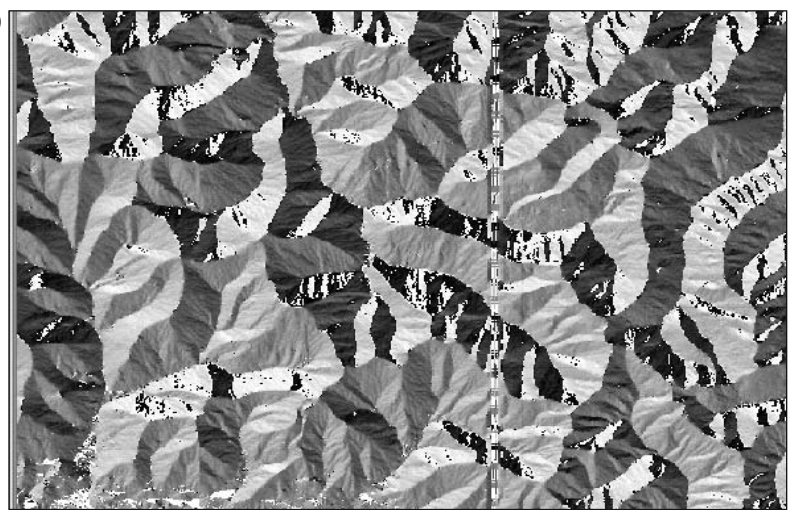

Figure 3. (a) Slope and (b) aspect of the study area.

terrain. The terrain slope and aspect image of the study area are shown in figure $3(\mathrm{a}-\mathrm{b})$. Slopes vary from $0^{\circ}-80^{\circ}$ with a mean value of $26.5^{\circ}$ and terrain aspect varies from $0^{\circ}-360^{\circ}$ having a southern trend with a mean value of $183.9^{\circ}$. Figure $4(\mathrm{a}-\mathrm{c})$ shows the IL image of the study area for topographic corrections and computed (Civco 1989; Kawata et al 1995; Riano et al 2003) using equation (3). The IL vary from -1 (low illumination) to +1 (high illumination) which is rescaled to a range of $0-255$ for the topographic models. The mean IL is estimated to be 176.8 (11 December 2005), 182.3 (18 January 2005) and 177.5 (21 February 2005).

\subsection{Estimation of coefficients for topographic models}

The results of different coefficient values $\left(c_{\lambda}, k(\lambda)\right.$ and $C_{\lambda}$ ) in equations (4), (5), (10), and (13) for three different date images of AWiFS are shown in table 2. It can be inferred that these coefficients are not same for different date images. These differences in the coefficients are attributed to different illumination condition, solar zenith and azimuth angle and temporal changes in snow surface physical properties. The results of Minneart constant show significantly different values for all the images between zero and one depending on the type of surface (Lambertian or non-Lambertian). The present results are attributed to non-Lambertian 
(a)

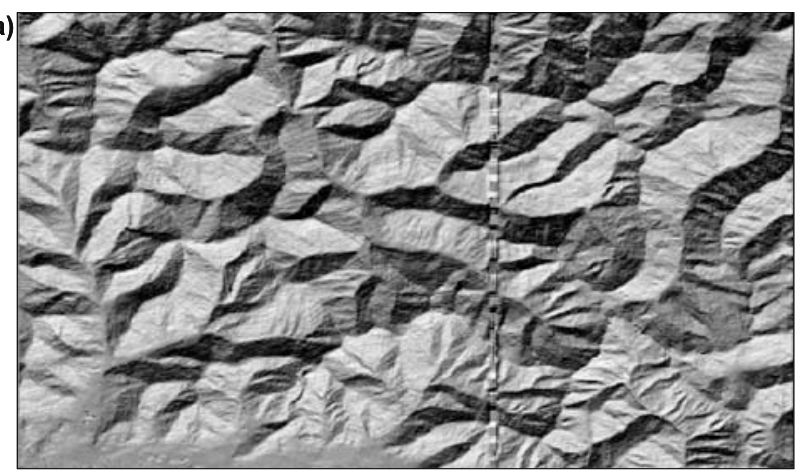

(b)

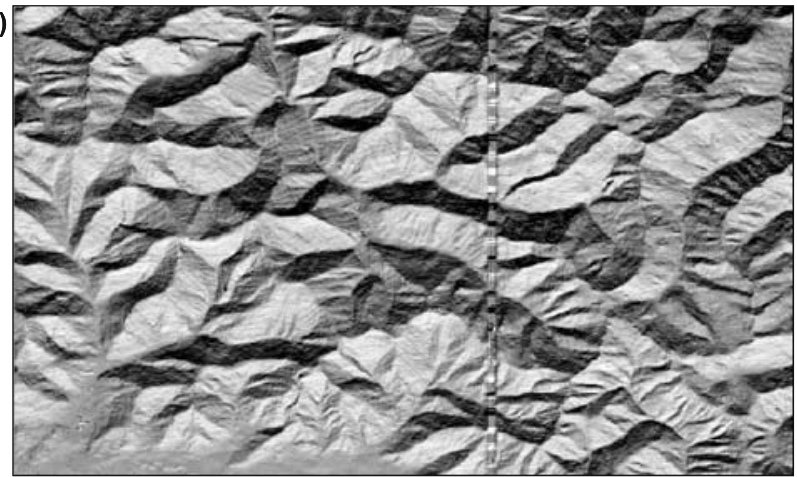

(c)

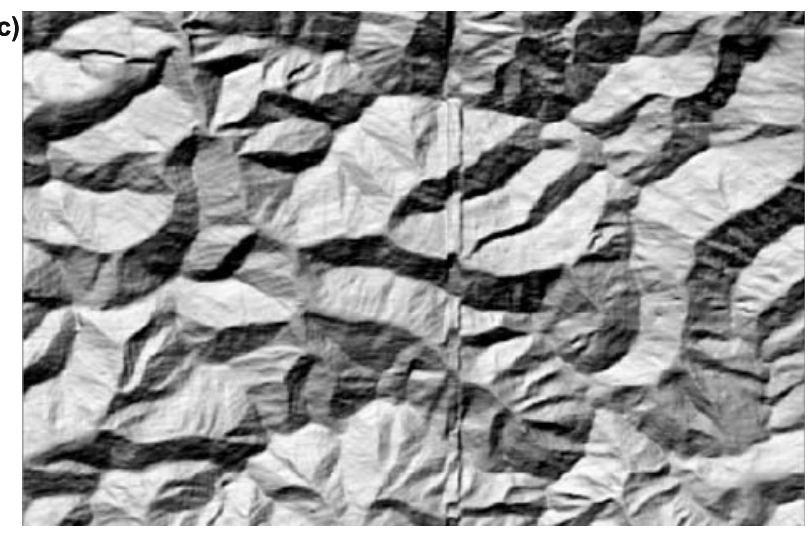

Figure 4. Illumination image of study area for AWiFS (a) 11 December 2005, (b) 18 January 2005 and (c) 21 February 2005.

surface as $k(\lambda)$ equal to one for perfect Lambertian surface and lies between zero and one for non-Lambertian surface. It can be inferred that Himalayan snow is non-Lambertian although most of the authors have assumed snow surface to be Lambertian in their reflectance model (Conese et al 1993; Richter 1997; Xin et al 2002; Srinivasulu and Kulkarni 2004).

\subsection{Application of topographic models in the Himalayan terrain and field results}

Figure 5 represents the false colour composite AWiFS image $(\mathrm{B} 2=$ blue, $\mathrm{B} 3=$ green and $\mathrm{B} 4=$ red) of 11 December 2005 (a) original uncorrected reflectance image (equation 1), (b) C-correction (equation 4), (c, d) Minneart correction (equations 5 and 7), (e) Civco's modified cosine corrections (equation 8), (f) Two stage normalization (equation 10) and (g) slope matching technique (equation 13). The results obtained using slope matching technique for other AWiFS images of January and February 2005 are shown in figure $6(\mathrm{a}-\mathrm{b})$. The visual analysis of the topographic results is compared qualitatively with the normalized difference snow index (NDSI) also. The NDSI is calculated using the following equation (Hall et al 1995):

$$
\mathrm{NDSI}=\frac{R_{\text {green }}-R_{\mathrm{SWIR}}}{R_{\text {green }}+R_{\mathrm{SWIR}}}
$$

where $R_{\text {green }}$ and $R_{\mathrm{SWIR}}$ are the reflectances in green (520-590 nm) and short wave infrared (1550-1700 nm) bands respectively. The NDSI estimated using equation (14) for all three AWiFS data are shown in figure $7(\mathrm{a}-\mathrm{c})$.

Figure $8(\mathrm{a}-\mathrm{b})$ shows the in-situ results of spectral characteristics of reflectance (average of multiple observations on snow surfaces) of pure snow measured in the field in open area without any interference from the surroundings in near real time for 18 January 2005 and exactly at the time of satellite pass on 21 February 2005. The FieldSpecFR Spectroradiometer obtained from M/S Analytical Spectral devices; USA in the wavelength range from $350 \mathrm{~nm}$ to $2500 \mathrm{~nm}$ was used to record the spectral reflectance of snow surface in field area. The sampling interval for the instrument is $1.4 \mathrm{~nm}$ for the region $350-1000 \mathrm{~nm}$ and $2 \mathrm{~nm}$ for the region $1000-2500 \mathrm{~nm}$. The fullwidth-half-maximum (FWHM) spectral resolution of the Spectroradiometer is $3 \mathrm{~nm}$ for the region $350-1000 \mathrm{~nm}$ and $10 \mathrm{~nm}$ for the region 1000-2500 nm. These field observations are used to validate the satellite estimated reflectance results in the AWiFS satellite bands using different topographic correction models. The quantitative results of satellite estimated reflectance values (for AWiFS of 18 January and 21 February 2005) using different topographic model with field validation (using in-situ observations) are given in table 3. The spectral reflectance of snow samples on the south and north aspects in one of the entire image of AWiFS of 21 February 2005 in band 2 (520-590 nm) for uncorrected and topographically corrected using slope matching method are shown in the figure $9(\mathrm{a}-\mathrm{d})$. The detailed quantitative analyses results of spectral reflectance for snow area in sunlit and shadow region using all topographic models are given in table 4 . Topographic corrections are also tested using a criteria based 
Table 2. Coefficients for different topographic normalization models.

\begin{tabular}{|c|c|c|c|c|}
\hline \multirow[b]{2}{*}{ Satellite images } & \multicolumn{4}{|c|}{ Coefficients } \\
\hline & C-correction & $\begin{array}{l}\text { Minneart } \\
\text { constant }\end{array}$ & $\begin{array}{c}\text { Two-stage } \\
\text { normalization } \\
\text { (mean } \\
\text { illumination) }\end{array}$ & $\begin{array}{c}\text { Slope match } \\
\text { method }\end{array}$ \\
\hline AWiFS - & 0.750 & 0.272 & 1.613 & 0.909 \\
\hline \multirow[t]{3}{*}{11 December 2005} & 0.661 & 0.310 & 2.184 & 0.981 \\
\hline & 0.630 & 0.330 & 3.040 & 1.032 \\
\hline & 0.410 & 0.370 & 3.932 & 0.693 \\
\hline AWiFS - & 0.535 & 0.250 & 2.36 & 0.835 \\
\hline \multirow[t]{3}{*}{18 January 2005} & 0.522 & 0.300 & 2.88 & 0.877 \\
\hline & 0.540 & 0.333 & 3.25 & 0.968 \\
\hline & 0.555 & 0.255 & 3.28 & 0.122 \\
\hline AWiFS - & 0.460 & 0.519 & 3.487 & 0.867 \\
\hline \multirow[t]{3}{*}{21 February 2005} & 0.447 & 0.539 & 3.709 & 0.891 \\
\hline & 0.473 & 0.522 & 4.039 & 0.939 \\
\hline & 0.380 & 0.498 & 3.233 & 0.212 \\
\hline
\end{tabular}
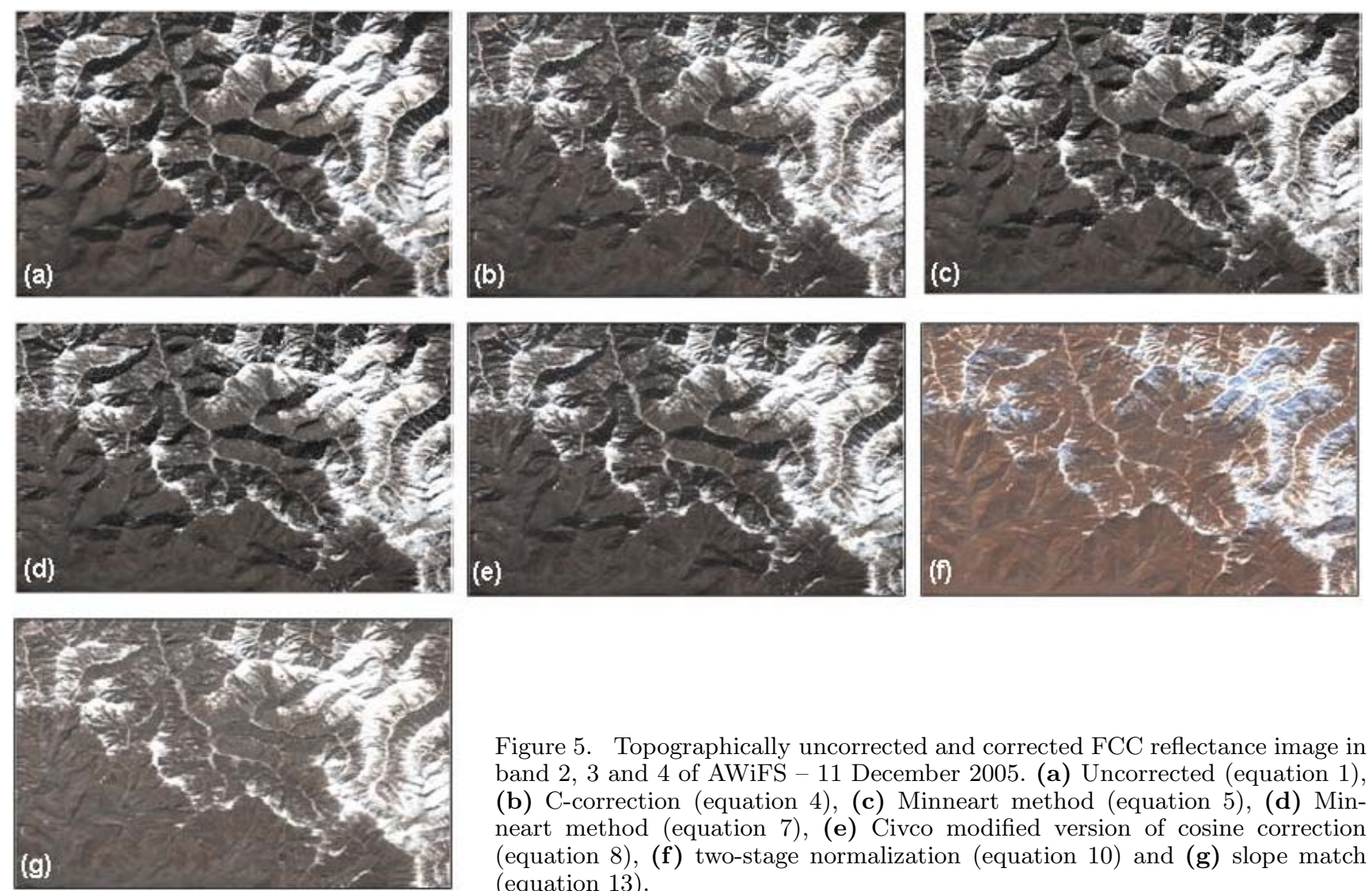

Figure 5. Topographically uncorrected and corrected FCC reflectance image in band 2, 3 and 4 of AWiFS - 11 December 2005. (a) Uncorrected (equation 1), (b) C-correction (equation 4), (c) Minneart method (equation 5), (d) Minneart method (equation 7), (e) Civco modified version of cosine correction (equation 8), (f) two-stage normalization (equation 10) and (g) slope match (equation 13).

on graphical analysis of $R_{T}$ (reflectance on the tilted surface) and $R_{H}$ (topographically corrected reflectance) versus IL for one image of 11 December 2005 proposed by (Meyer et al 1993; Ekstrand 1996) various authors as shown in figure 10.

\section{Discussion}

\subsection{Visual analysis (qualitative analysis)}

The visual interpretation of the false color composite images reveals that occlusions due to 

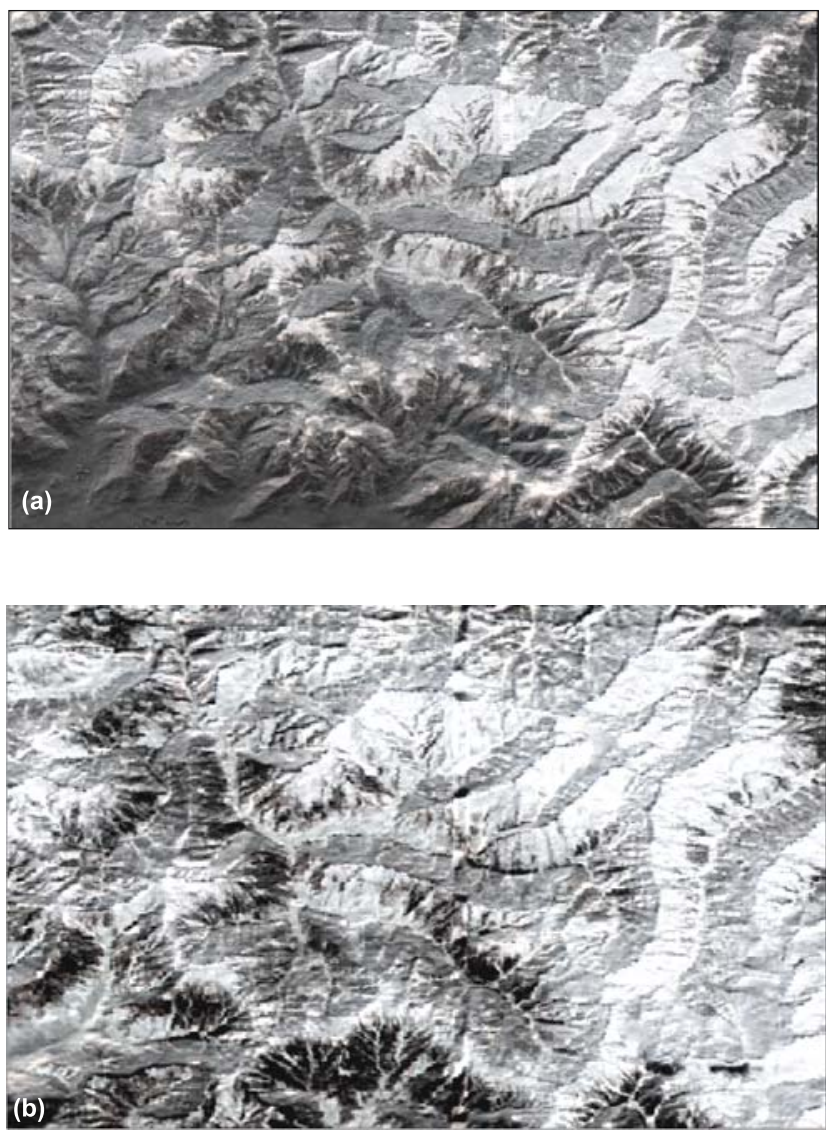

Figure 6. Topographically corrected AWiFS images using slope matching method; (a) 18 January 2005 and (b) 21 February 2005.

terrain illumination effects in the original image after topographic corrections are still pronounced except two-stage normalization and slope matching. Area of slopes that face away from the Sun are still darker and three dimensional relief impression is not suppressed completely (figure 5a-e). However, visual analysis of the results obtained by slope matching method looks more realistic as compared to two-stage normalization, which shows very high brightness on the sunny and shady slopes (figure 5f, g). Although, corrected images using both these methods show flat view of the terrain, and the three dimensional relief effects is minimized. The visual analysis by slope matching also reveals that there is less variability in spectral response of the snow classes and can handle the corrections for the problem of shaded relief very well, where the incident angle $i$ is $\geq 90^{\circ}$. The similar interpretation for visual analysis are also reported by (Law and Nichol 2004; Nichol 2006) for high resolution IKONOS data. The topographically corrected results are compared visually with NDSI images (figure $7 \mathrm{a}-\mathrm{c}$ ) also. NDSI is used to monitor snow cover information under mountain shadow and it can very well identify snow
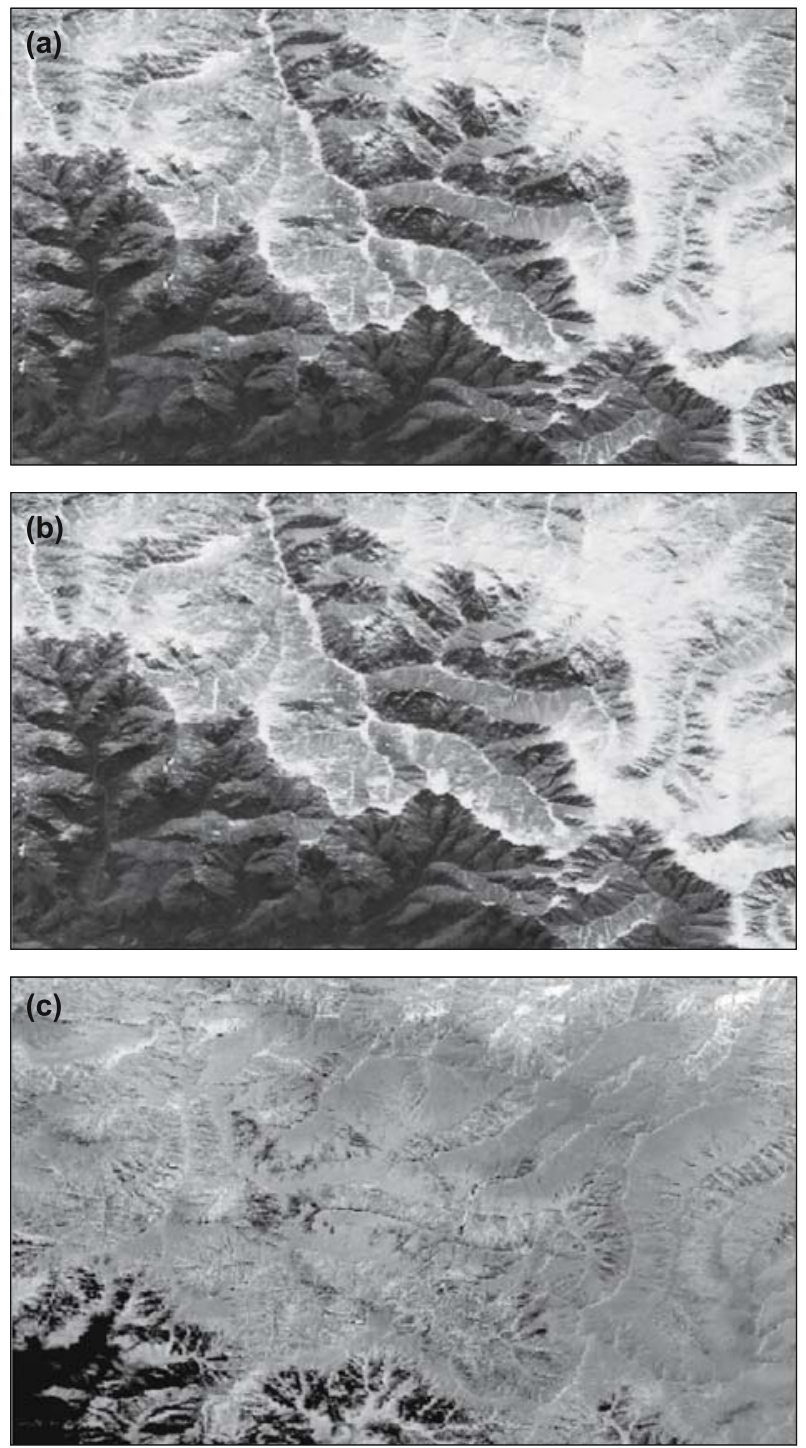

Figure 7. NDSI images of the study area (a) 11 December 2005, (b) 18 January 2005, and (c) 21 February 2005.

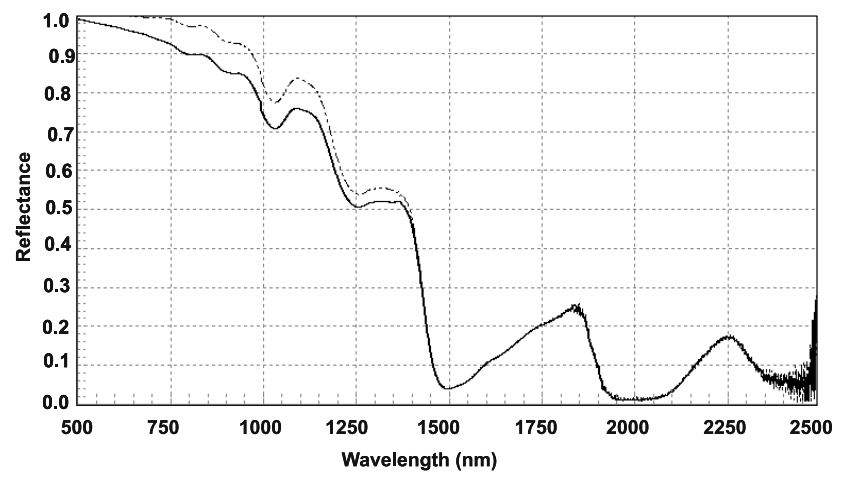

Figure 8. In-situ observations of spectral reflectance characteristics of pure snow (a) 18 January 2005 and (b) 21 February 2005.

in shadow areas. The visual comparative analysis of topographically corrected results by slope matching method (figures $5 \mathrm{~g}$ and $6 \mathrm{a}-\mathrm{b}$ ) with NDSI 
images (figure $7 \mathrm{a}-\mathrm{c}$ ) show that only this method gives most acceptable results and retrieve the actual land cover information for low illumination areas $(\cos i \leq 0)$ and other methods fail to produce successful results.

\subsection{Quantitative analysis}

\subsubsection{Model validation}

The results obtained using different topographic models are compared with the in-situ observations of spectral reflectance recorded at the time of satellite pass at Indian standard time (1100 IST) on 18 January 2005 and 21 February 2005. The field observations for 11 December 2005 were not available, therefore topographic results are compared only for two AWiFS images of January and February 2005. The field observations are collected at number of points for pure snow samples and the mean of all the observations are estimated (figure 8a-b) at a latitude of $34.622^{\circ}$ and longitude of $74.425^{\circ}$. As the surrounding field area has coniferous trees, it is very difficult to verify the field reflectance results accurately at the same latitude and longitude with the satellite estimated value because of these objects, which may give some variations in the reflectance values of medium spatial resolution pixel of $56 \mathrm{~m}$. Therefore, the satellite estimated reflectance was verified with the field results in the neighbourhood pure pixel of snow on the image. It is observed from figure $8(\mathrm{a}-\mathrm{b})$ that reflectance of snow is high at the red end of the visible spectrum. It tends to decline in the near-infrared region until $1050 \mathrm{~nm}$, where slight gain in reflectance occurs and gives a minor peak at approximately $1090 \mathrm{~nm}$ to $1100 \mathrm{~nm}$. The reflectance also shows minor peaks around $1800 \mathrm{~nm}$ and $2250 \mathrm{~nm}$ with a sharp depression of reflectance around $1950 \mathrm{~nm}$ to $2050 \mathrm{~nm}$. This sharp depression is due to strong water absorption bands at these wavelengths. The comparative analysis of different topographic results with in-situ observations in table 3 shows that all the topographic models underestimate the reflectance in different satellite bands of AWiFS except slope matching technique which gives most acceptable results with high accuracy $(>96 \%)$ and very low relative error (0.2-0.4) for both AWiFS images of January and February 2005.

\subsubsection{Performance of topographic corrections}

The performance of the topographic corrections can be assessed quantitatively using several

Table 3. Validation of topographic normalization models with field results of spectral reflectance using spectroradiometer.

\begin{tabular}{|c|c|c|c|c|}
\hline \multirow[b]{2}{*}{$\begin{array}{l}\text { Topographic } \\
\text { models }\end{array}$} & \multicolumn{4}{|c|}{ Spectral reflectances in AWiFS bands } \\
\hline & $\begin{array}{c}\text { B2: } \begin{array}{c}520-590 \\
(\mathrm{~nm})\end{array}\end{array}$ & $\begin{array}{c}\text { B3: } 620-680 \\
(\mathrm{~nm})\end{array}$ & $\begin{array}{c}\text { B4: } 770-860 \\
(\mathrm{~nm})\end{array}$ & 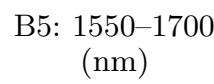 \\
\hline \multicolumn{5}{|c|}{$\begin{array}{l}\text { AWiFS - Date: } 18 \text { January } 2005 \text {, pixel location: latitude }\left(34.626^{\circ}\right) \text { and longitude }\left(74.416^{\circ}\right) \text {. Slope: } \\
9.4^{\circ} \text { and aspect: south }\left(180^{\circ}\right)\end{array}$} \\
\hline Original image & 0.947 & 0.977 & 1 & 0.135 \\
\hline C-correction (equation 4) & 0.841 & 0.854 & 0.874 & 0.120 \\
\hline Minneart (equation 5) & 0.877 & 0.908 & 0.926 & 0.133 \\
\hline Minneart (equation 7) & 0.865 & 0.893 & 0.914 & 0.124 \\
\hline Civco (equation 8) & 0.761 & 0.786 & 0.804 & 0.109 \\
\hline Two stage (equation 10) & 0.426 & 0.322 & 0.243 & 0.032 \\
\hline Slope match (equation 13 ) & 0.989 & 1 & 1 & 0.141 \\
\hline Field results & 1 & 1 & 0.96 & 0.132 \\
\hline \multicolumn{5}{|c|}{$\begin{array}{l}\text { AWiFS - date: } 21 \text { February } 2005 \text {, pixel location: latitude }\left(34.624^{\circ}\right) \text { and longitude }\left(74.421^{\circ}\right) \text {. Slope: } \\
11.3^{\circ} \text { and aspect: south }\left(165^{\circ}\right)\end{array}$} \\
\hline Original image & 0.974 & 0.958 & 0.948 & 0.126 \\
\hline C-correction (equation 4) & 0.862 & 0.854 & 0.857 & 0.113 \\
\hline Minneart (equation 5) & 0.891 & 0.874 & 0.866 & 0.116 \\
\hline Minneart (equation 7) & 0.882 & 0.866 & 0.858 & 0.115 \\
\hline Civco (equation 8) & 0.751 & 0.740 & 0.731 & 0.098 \\
\hline Two stage (equation 10) & 0.580 & 0.532 & 0.521 & 0.045 \\
\hline Slope match (equation 13) & 1 & 0.993 & 0.984 & 0.131 \\
\hline Field results & 0.978 & 0.957 & 0.90 & 0.135 \\
\hline
\end{tabular}



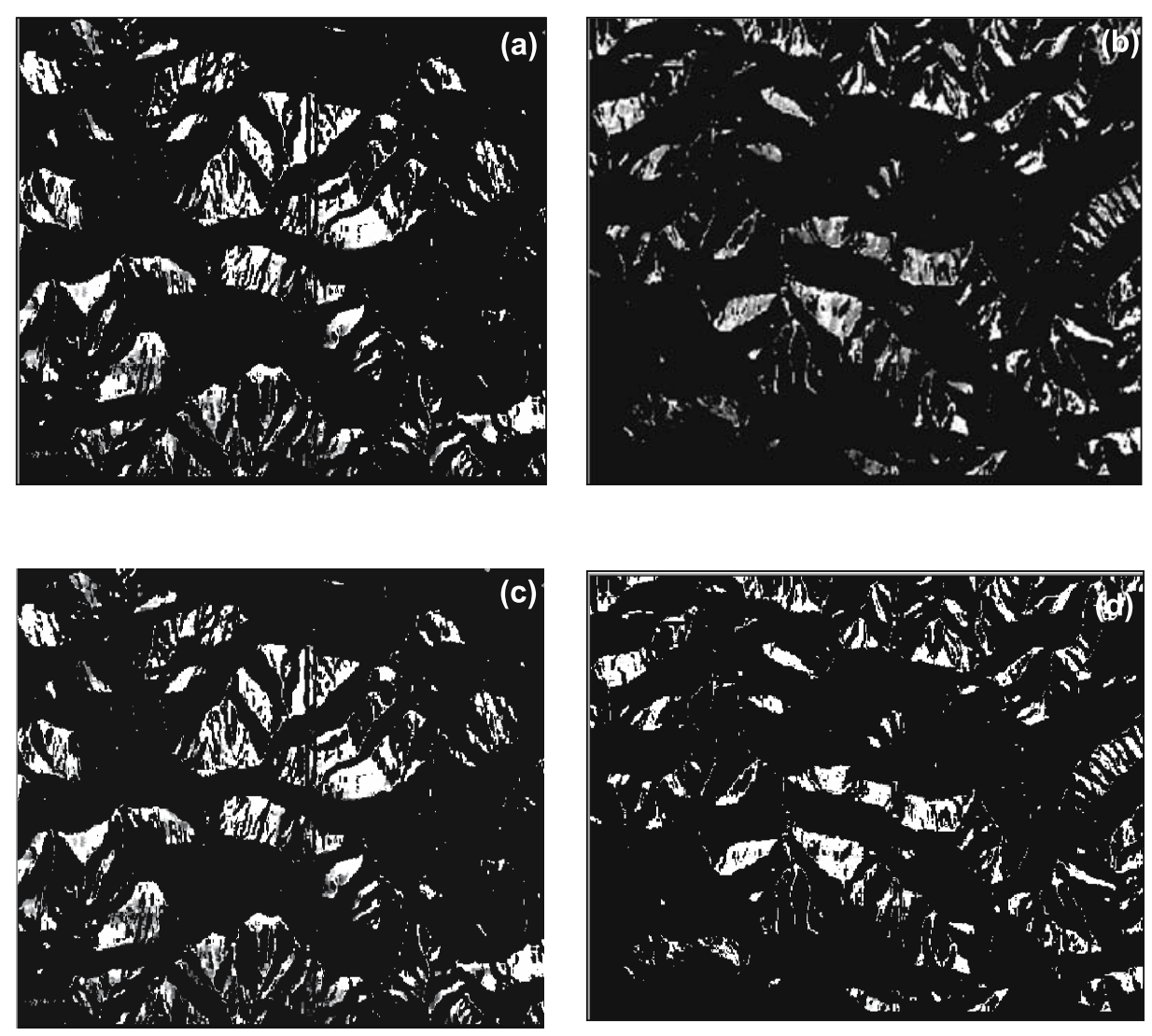

Figure 9. Snow samples on the south and north aspects for AWiFS images of 21 February 2005 (a) south aspect before topographic corrections, (b) north aspect before topographic corrections, (c) south aspect after topographic corrections using slope matching and (d) north aspect after topographic corrections using slope matching.

possible methods (Riano et al 2003). First method is based on the analysis of changes in spectral characteristics of the training snow samples on the south and north aspects after the topographic corrections (Civco 1989). Second criterion is based on the graphical analysis of $R_{\lambda T}$ and $R_{\lambda H}$ with illumination, IL (Meyer et al 1993; Ekstrand 1996). The spectral reflectance on the tilted surface, $R_{\lambda T}$, varies with illumination angle before topographic corrections and should remain constant after the corrections have been carried out in satellite data. Both these methods are implemented to test the topographic corrections by different methods. The analysis of changes in the spectral reflectance characteristics of the image is tested before and after the topographic corrections choosing snow samples on south and north aspects for all the entire images. Training areas of snow were created on both sunny (south aspect) and shady slopes (north aspect) using normalized difference snow index (NDSI $\geq 0.6)$ in figure $9(\mathrm{a}-\mathrm{d})$. Mean values of reflectance on the sunny slopes are expected to decrease while those on the shady side are expected to increase for successful topographic corrections (Law and Nichol 2004). It can be inferred from the quantitative analysis in the table 4 that only slope matching method is able to retrieve acceptable values of spectral reflectance of snow samples in shadow areas. It is also observed that this method is applicable to different solar geometry, e.g., varying solar zenith angle and differential illumination condition. Slope matching method is found to be unique and gives the best results (satisfy all the four criteria) for qualitative and quantitative analysis as compared to other methods. Although the values of reflectance decreases on the south aspect and increases on the north aspect by all the methods but the values calculated on the north aspect does not represent to true snow reflectance values when compared with the results of slope matching method. The plot of mean $R_{\lambda H}$ (in band 2) with mean IL after the topographic corrections (figure 10a-f) is observed to be invariant by all the methods but quantitative assessment as discussed above fails except for slope matching. The slope matching method is unique among all the methods and is more suitable for Himalayan terrain for qualitative and quantitative assessment for snow cover analysis. 


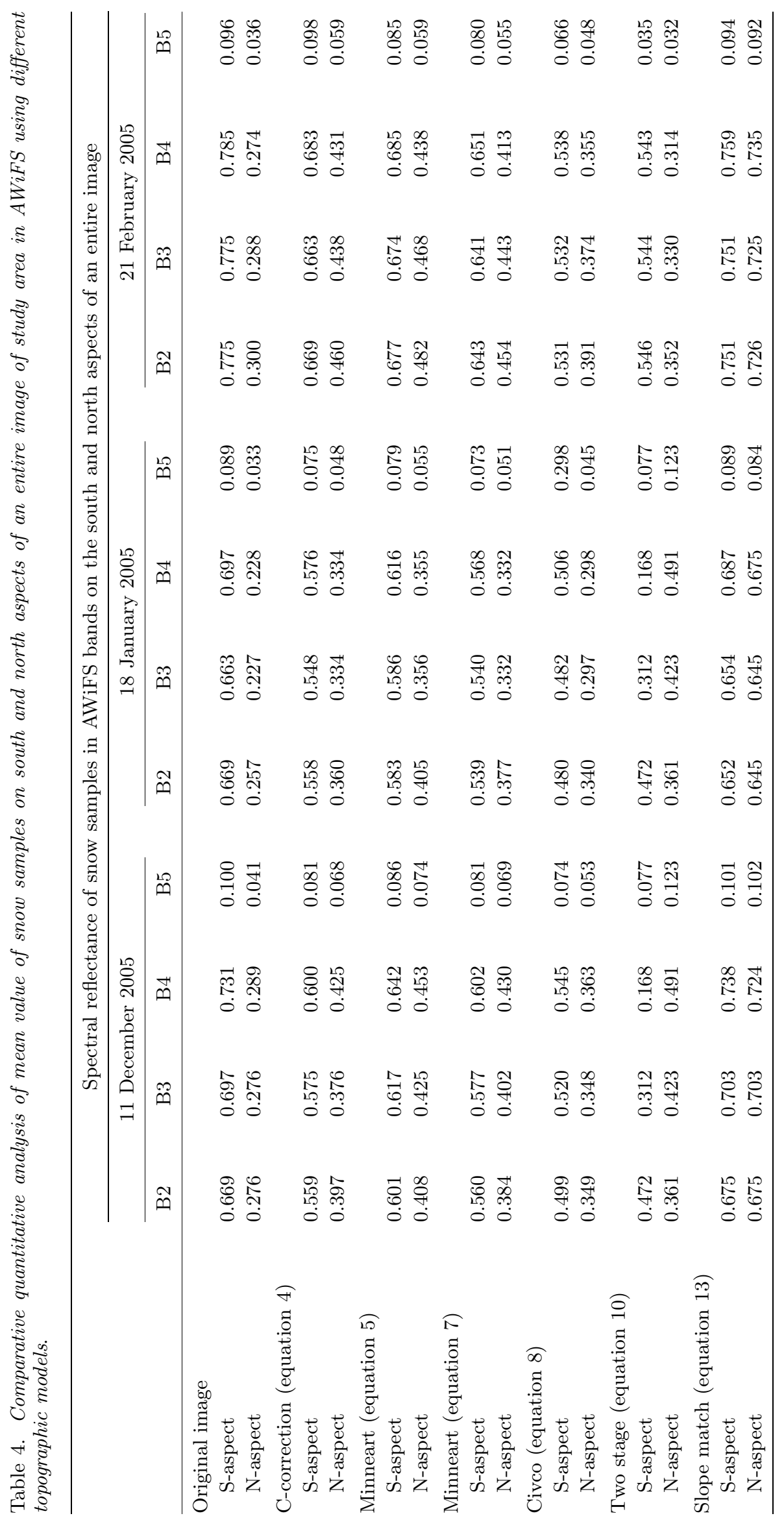



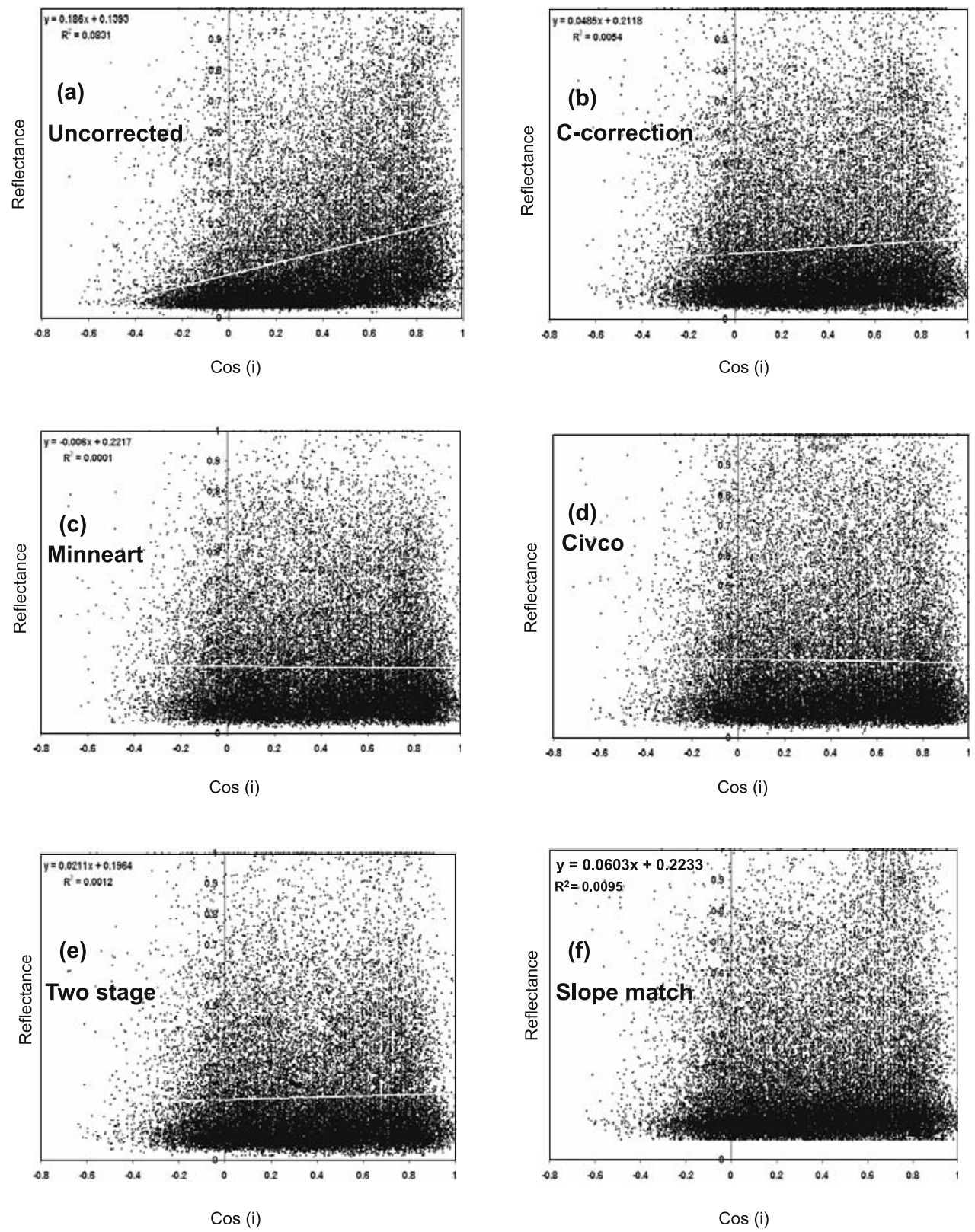

Figure 10. Graphical analysis of reflectance in AWiFS band $2(520-590 \mathrm{~nm})$ versus illimunation (IL) before and after the topographic corrections for AWiFS image of 11 December 2005 image. (a) Without topographic corrections, (b) C-correction, (c) Minneart (equation 5), (d) Civco modified version of cosine correction, (e) two-stage normalization and (f) slope matching method.

\section{Conclusions}

Qualitative and quantitative comparative analysis of different topographic models for Himalayan terrain is reported in this paper. The effect of terrain topography on the satellite estimated snow reflectance is illustrated by using AWiFS images of three different dates with and without snow cover in shadow areas. All the correction methods are verified using four criteria, i.e.,

- visual analysis

- model validation with in-situ observations
- reflectance of training snow samples on the south and north aspects before and after topographic corrections and

- graphical analysis.

Visual analysis reveals that two-stage normalization and slope matching methods are suitable for Himalayan terrain. Graphical analysis also satisfies the condition for all the methods and validates suitability for Himalayan region. Model validation with field results and spectral reflectances of snow samples on the south and north aspects fails for all the methods except slope matching. It is concluded 
that no model can be suitable for Himalayan region just satisfying only one or two criteria. Slope matching technique is the only method which satisfies all the four criteria successfully. It is found to be unique and most suitable for Himalayan terrain for qualitative and quantitative analysis of snow cover and also for varying solar geometry. Another important conclusion drawn in this paper is that snow is highly non-Lambertian surface as Minneart constant is estimated to lie between zero and 0.5. Topographic corrections are very useful for further applications as sub-pixel snow mapping, energy balance studies, climatic modeling, snow melt run-off modeling, avalanche hazard analysis, change detection analysis, etc.

\section{Acknowledgements}

The authors would like to thank Dr R N Sarwade, Director SASE and Dr A V Kulkarni, Scientist "SG", Space Application Centre for their encouragement, suggestions and comments. Thanks are also due to two anonymous referees for their valuable suggestions to improve the revised version of the paper.

\section{References}

Brun E, Martin E, Simon V, Gendre C and Coleou C 1989 An energy and mass model of snow cover suitable for operational avalanche forecasting; J. Glaciol. 15(121) 333-341.

Chavez P S Jr 1989 Radiometric calibration of Landsat Thematic Mapper multispectral images; Photogramm. Eng. Remote Sens. 55 1285-1294.

Chavez P S Jr 1996 Image-based atmospheric corrections revisited and improved; Photogramm. Eng. Remote Sens. 62 1025-1036.

Civco D L 1989 Topographic normalization of Landsat Thematic Mapper digital imagery; Photogramm. Eng. Remote Sens. 55 1303-1309.

Colby J D 1991 Topographic normalization in rugged terrain; Photogramm. Eng. Remote Sens. 57 531-537.

Conese C, Gilabert M A, Maselli F and Bottai L 1993 Topographic normalization of TM scenes thorough the use of an atmospheric corrections method and digital terrain models; Photogramm. Eng. Remote Sens. 59 1745-1753.

Davis R E, Nolin A W, Jordan R and Dozier J 1993 Towards predicting temporal changes of the spectral signature of snow in visible and near-infrared wavelengths; J. Glaciol. 17 143-147.

Deems J S 2001 Topographic controls on spatial and temporal variation in snow temperatures in a mountain snowpack; Proceesing of the 69th Western Snow Conference Sun Valley ID 131-134.

Dozier J 1984 Snow reflectance from Landsat Thematic Mapper; IEEE Trans. Geosci. Remote Sens. 22(3) $323-328$.

Duguay C R and LeDrew E F 1991 Mapping surface albedo in the East slope of the Colorado front range, U.S.A., with Landsat Thematic Mapper; Arct. Alps. Res. 23(2) 213-223.
Duguay C R and LeDrew E F 1992 Estimating surface reflectance and albedo from Landsat-5 TM over rugged terrain; Photogramm. Eng. Remote Sens. 58 551-558.

Duguay C R 1993 Modelling the radiation budget of alpine snowfields with remotely sensed data: model formulation and validation; Ann. Glaciol. 17 288-293.

Ekstrand S 1996 Landsat-TM based forest damage assessment: Correction for topographic effects; Photogramm. Eng. Remote Sens. 62 151-161.

Hall D K, Chang A T C and Siddalingaiah H 1988 Reflectances of glaciers as calculated using Landsat-5 Thematic Mapper data; Remote Sens. Environ. 25 311-321.

Hall D K, Riggs G A and Salomonson V V 1995 Development of methods for mapping global snow cover using Moderate Resolution Imaging Spectroradiometer Data; Remote Sens. Environ. 54 127-140.

Haertel V F and Shimabukuro Y E 2005 Spectral linear mixing model in low spatial resolution image data; IEEE Trans. Geosci. Remote Sens. 43(11) 2555-2562.

Holben B and Justice C 1980 The topographic effect on spectral response from Nadir pointing sources; Photogramm. Eng. Remote Sens. 46 1191-1200.

Kasten F 1962 Table of solar altitudes for geographical latitudes; CRREL Special Report, 57 U.S. Army Corps of Engineers, Hanover, New Hampshire.

Kawata Y, Ueno S and Ohtani A 1995 The surface albedo retrieval of mountainous forest area from satellite MSS data; Appl. Math. Comput. 69 41-59.

Law K H and Nichol J 2004 Topographic correction for differential illumination effects on IKONOS satellite imagery; Proceeding of XXth ISPRS symposium.

Leprieur C, Durand J M and Peyron J L 1988 Influence of topography on forest reflectance using Landsat Thematic Mapper and digital terrain data; Photogramm. Eng. Remote Sens. 54 491-496.

Meyer P, Itten K I, Kellenbenberger T, Sandmeier S and Sandmeier R 1993 Radiometric corrections of topographically induced effects on Landsat TM data in an alpine environment; ISPRS J. Photogramm. Remote Sens. 48 17-28.

Minneart M 1941 The reciprocity principle in lunar photometry; J. Astrophys. 93 403-410.

Mishra V D, Negi H S, Rawat A K, Chaturvedi A and Singh R P 2008 Retrieval of sub-pixel snow covered Himalayan region using medium and coarse resolution remote sensing data; Int. J. Remote Sens. (in press).

Mukkoth V N 2004 Snow melt runoff modeling using MODIS in Elaho River Basin, British Columbia; Environmental Information Archieves 2 526-530.

Nichol J, Hang L K and Sing W M 2006 Empirical correction of low sun angle images in steeply sloping terrain: a slope matching technique; Int. J. Remote Sens. 27(3-4) 629-635.

Pandya M R, Singh R P, Murali K R, Babu P N, Kirankumar A S and Dadhwal V K 2002 Bandpass solar exo-atmospheric irradiance and Rayleigh optical thickness of sensors on board Indian Remote Sensing Satellites - 1B, -1C, -1D, and P4; IEEE Trans. Geosci. Remote Sens. 40(3) 714-717.

Riano D, Chuvieco E, Salas J and Aguado I 2003 Assessment of different topographic corrections in Landsat-TM data for mapping vegetation types; IEEE Trans. Geosci. Remote Sens. 41(5) 1056-1061.

Richter R 1997 Correction of atmospheric and topographic effects for high spatial resolution satellite imagery; Int. J. Remote Sens. 18 1099-1111.

Romanov P, Tarpley D, Gutman G and Carroll 2003 Mapping and monitoring snow-cover fraction over North America; J. Geophys. Res. 1088619. 
Smith J A, Lin T L and Ranson K J 1980 The Lambertian Assumption and Landsat Data; Photogramm. Eng. Remote Sens. 46(9) 1183-1189.

Song J and Gao W 1999 An improved method to derive surface albedo from narrowband AVHRR satellite data: narrowband to broadband conversion; J. Applied Meteorol. 38 239-249.

Song C, Woodcock C E, Seto K C, Lenney M P and Macomber A S 2001 Classification and change detection using Landsat TM data: when and how to correct atmospheric effects; Remote Sens. Environ. 75 230-244.

Srinivasulu J and Kulkarni A V 2004 Estimation of spectral reflectance of snow from IRS-1D LISS-III sensor over the Himalayan terrain; Proc. Indian Acad. Sci. (Earth Planet. Sci.) 113 117-128.
Teillet P M, Guindon B and Goodenough D G 1982 On the slope aspect correction of multispectral scanner data; Canad. J. Remote Sens. 8 84-106.

Tokola T, Sarkeala J and Van der Linden M 2001 Use of topographic correction in Landsat TM-based forest interpretation in Nepal; Int. J. Remote Sens 22 551-563.

Upadhyay D S 1995 Snow melt processes; Cold Climate Hydrometeorology. New Age Int. Pvt. Ltd. Publ., 213-230.

Van Der Meer F 1996 Spectral mixture modeling and spectral stratigraphy in carbonate lithofacies mapping; ISPRS J. Photogramm. Remote Sens. 51 150-162.

Xin L, Koike T and Guodong C 2002 Retrieval of snow reflectance from Landsat data in rugged terrain; Ann. Glaciol. 34 31-36.

MS received 28 January 2008; revised 14 October 2008; accepted 16 October 2008 